\title{
Continuous Hospitality with Social Robots at a hotel
}

\author{
Junya Nakanishi $^{1}$ (D) Itaru Kuramoto ${ }^{2} \cdot$ Jun Baba $^{3} \cdot$ Kohei Ogawa $^{1} \cdot$ Yuichiro Yoshikawa ${ }^{1} \cdot$ Hiroshi Ishiguro $^{1}$
}

Received: 6 June 2019 / Accepted: 5 February 2020 / Published online: 20 February 2020

(c) Springer Nature Switzerland AG 2020

\begin{abstract}
Social robots are being increasingly employed in service encounters at hotels. This study explored the possibility that social robots can engage in heartwarming interactions with hotel customers. A collaboration design known as 'Continuous Hospitality with Social Robots', in which social robots compensate for gaps in hospitality through heartwarming interaction, was evaluated. A field test was conducted in which social robots engaged in heartwarming interaction with customers in a public area of a hotel and then collected customers' impressions of the social robots and overall service via a questionnaire and an interview. The results demonstrate social robots' potential for engaging in heartwarming interactions that enhance overall customer satisfaction through the use of the 'Continuous Hospitality with Social Robots' collaboration design. An exploratory analysis suggests that the perceived impressions of the interaction with social robots are influenced by customer gender and the duration of interactions. Furthermore, the results suggest that social robots could be utilized in other roles at hotels, namely effective advertisement through heartwarming interaction and mental support for employees who do not interact with customers.
\end{abstract}

Keywords Social robots $\cdot$ Heartwarming interaction $\cdot$ Hotels $\cdot$ Hospitality $\cdot$ Collaboration with robots

\section{Introduction}

\subsection{Background and issue}

Social robots are being increasingly employed in service encounters at hotels $[15,38]$. For example, Henn-na Hotel is said to be the first robot-staffed hotel, where social robots engage with customers at the front desk and act as in-room assistants. Aloft Hotels employ robotic butlers which navigate the hotel and deliver requested items to customer rooms. Moreover, many studies have tested the performance of various social robots in service encounters (e.g. at information desks $[24,32,40]$, information broadcasting [26, 27], bellboy tasks [28], and customer feedback gathering [7]).

In service encounters, heartwarming interaction is required for hospitality. Heartwarming interaction occurs when an employee's behaviour and attitude create feelings of interpersonal warmth between the employee and their customers. For instance, an employee welcomes a customer with a smile and a sincere greeting, always pays careful attention to a customer, and properly asks if there is anything else an employee could help him/her with. Such interaction is categorized as hospitality or 'omotenashi' (Japanese-style hospitality) [23, 31]. This heartwarming interaction-specifically, hospitality-is important in hotel service because such interactions enhance customer satisfaction in the whole hotel service [16], consequently increasing the number of repeat customers and leading to positive word-of-mouth [22].

Here, a question arises: can a social robot engage in heartwarming interaction at a hotel? Some studies mention the effects of heartwarming interaction with robots (e.g. smiling [8], greeting [36, 37]). Although these studies show the possibility that social robots can engage in heartwarming interactions, they did not analyse customer

Junya Nakanishi, nakanishi@irl.sys.es.osaka-u.ac.jp | ${ }^{1}$ Osaka University, 1-1, Machikaneyama, Toyonaka, Osaka, Japan. ${ }^{2}$ The University of Fukuchiyama, Ichihori, Fukuchiyama, Kyoto 3370, Japan. ${ }^{3}$ Cyber Agent Inc., 12-1, Dogenzaka, Tokyo, Japan. 
reactions in practical hotel situations. It is thus unclear whether heartwarming interaction with robots will contribute to hospitality in hotels because interactions are affected by the surrounding environment and the situational context [33]. Thus, a field test is required in order to establish a connection between robot technology and the service industry. Moreover, the manner in which humans and robots collaborate in hotel service should be considered [25] because the contribution of tools depends on how they are used. Therefore, designing methods for collaboration with robots and subsequently testing them in practical situations is important.

This paper proposes the collaboration design 'Continuous Hospitality with Social Robots' and reports on a field study conducted at a hotel to evaluate the design. The design aims to offer interpersonal warmth to customers as often as possible in order to enhance hospitality. For this purpose, social robots compensate for gaps in hospitality; that is, robots are assigned to serve in situations in which a human employee typically does not serve a customer. In this field study, a trial was conducted in which social robots engaged in heartwarming interactions with customers in public areas of a hotel (i.e. a corridor and an elevator hall) throughout the day. To investigate the impact of this heartwarming interaction with social robots, customer impressions of these interactions were collected via a questionnaire and an interview. In addition, differences stemming from personal and situational factors were also highlighted in the analysis. Moreover, other applications of the proposed system were explored, namely effective advertising through heartwarming interaction.

\subsection{Research questions addressed by this study}

A field study was conducted to evaluate the collaboration design 'Continuous Hospitality with Social Robots'. During the evaluation, the following questions were addressed:

Q1 Can interaction with social robots create interpersonal warmth in customers in a hotel environment?

Q2 Can robots compensating for gaps in hospitality contribute to customer satisfaction in the whole hotel service?

Previous studies have shown that impressions about interactions with social robots are affected by various factors (e.g. the number of robots $[26,27]$, gender differences [8]). Similarly, in practical situations various factors may affect the impression of the interaction. Understanding these factors is essential for determining the optimal form of heartwarming interaction with social robots. Thus, an exploratory investigation was conducted with focus on the following questions:
Q3 Does the number of robots (i.e. one vs. two robots), the gender of the customer, or the fact that the customer is alone or in a group affect impressions of heartwarming interactions?

Q4 Is an impression of heartwarming interaction affected by the number of nights stayed, frequency of use of a hotel, or the amount of time spent interacting with a social robot or a voice-controlled speaker?

In addition, heartwarming interaction may offer a good opportunity to advertise. Thus, customer reactions to a social robot's recommendations during a heartwarming interaction were investigated by posing the following question:

Q5 Does a customer follow recommendations from a social robot engaging in a heartwarming interaction?

To answer the above questions, a prototype of a heartwarming interaction system employing social robots was developed and introduced it into a hotel.

\section{Literature review}

\subsection{Social robots in a hotel}

Various robots have already been employed in service encounters in hotels $[15,38]$, and researchers have investigated using social robots for more complex tasks involving human-robot interactions in hotel environments. For instance, a long-term assessment with a humanoid robot was conducted to test and improve robots' ability to perform bellboy tasks [28]. In the experiment, bellboy tasks involved guiding customers, providing useful information, and providing other hotel-related services. Using robots to gather customer feedback about hotel service was tested with an autonomous mobile robot [7]. Other studies tested humanoid or animal-like robots engaging in information desk tasks in lobbies or customer rooms [24, 32, 40]. In the latter study, Rodriguez-Lizundia et al. focused on the robots' appearance and behaviour (i.e. with/without robotic body, awake/asleep, and robot/user starts communication) and assessed their effect on the distance, duration, and frequency of communication between robots and customers. In particular, Pan et al. assessed how the number of robots affected the attraction of attention in information broadcasting tasks [26, 27]. While the above studies revealed some technical aspects of robotic design and examined the performance of those designs in service encounter tasks, several researchers suggest that the customer's interactive experience (e.g. perception and mind) 
with a robot should receive greater focus in future research as well $[29,39]$. It is believed that heartwarming interaction is one of the most important aspects in human-robot interaction research related to hotel service.

\subsection{Heartwarming interaction and social robots}

In this analysis, heartwarming interaction is defined as behaviour and attitude which create feelings of interpersonal warmth. Interpersonal warmth is a positive, mild, volatile emotion involving physiological arousal induced by the communication partner's behaviour and attitude $[1,4]$, and relates to perceived intent, including friendliness, helpfulness, sincerity, trustworthiness, and morality [11]. Management research on hospitality in the service industry has focused on heartwarming interaction [19, $20,35]$ because it enhances customer satisfaction with hotel service [16] and consequently increases the number of frequent customers and leads to positive word-ofmouth [22]. In service encounters, heartwarming interaction includes a smile, a greeting, and eye contact $[2,4,34]$. Such interaction is categorized as hospitality or 'omotenashi' (Japanese-style hospitality) $[23,31]$ and is regarded as emotional labour [21] in the service industry.

In recent years, as social robots have been introduced into encounters in the service industry, the effects of robots' heartwarming interactions have begun to receive attention from some researchers. Chung-En assessed how smiles from a humanoid robot and a human affect customer perceptions in service encounters through a vignette experiment, a method to explain a hypothetical situation and draw participants' perceptions or impressions [8]. It was shown that no statistically significant difference exists between a humanoid robot and a human in terms of feelings of interpersonal warmth. This suggests that social robots can create feelings of interpersonal warmth as effectively as a human employee. In contrast, a laboratory experiment showed that a humanoid robot's greeting to a human is ignored more often than a teleoperated greeting [36] or a human greeting [37]. This result implies that the robot's greeting could not have enough opportunities to create feelings of interpersonal warmth as effectively as a human's greeting. However, these studies do not reveal how heartwarming interactions affect customers in practical hotel situations.

\subsection{Collaborative service design for social robots in a hotel}

The study examined collaboration with robots in a hotel in which various robots are present [25]. The first form of collaboration involves using robots as a replacement for human labour. Actually, most studies have developed social robots capable of engaging in tasks typically performed by humans $[24,28,32,40]$, implying an intention to replace human labour with social robots. Hennna hotel advocates replacement of human labour with robots for ultimate efficiency $[15,25]$. The hotel measured efficiency of labour in terms of time and cost and then assigned humans and robots to each service task in order to improve efficiency as much as possible. On the other hand, researchers anticipated that there would be barriers to these collaborations, including resistance from employees and customers [14]. For example, employees may fear hotel staffing reductions resulting from the labour replacement.

The second form of collaboration involves using robots as a tool to extend hotel service, with the aim of increasing the appeal of a hotel through robotic support [14]. In general, robots are free from the limitations of human labour and human nature. For instance, robots can work without breaks and perform very tedious tasks many times without complaints or forgetting to do them. Thus, robots can be assigned to tasks which are usually not performed by human employees. By doing this, a hotel can offer continuous service to customers during their entire hotel stay. This form of collaboration could allow a hotel to increase service quality because gaps in service are reduced. Moreover, the form does not take the jobs of human employees. Therefore, this form of collaboration would be acceptable in most hotels as the first step in introducing robotic labour. This field study evaluated this collaboration form.

\section{Method}

\subsection{Heartwarming interaction system employing social robots in hotels}

This heartwarming interaction system focuses on warm words and informing actions as the basis for heartwarming interactions. The warm words action involves greeting, welcoming, and saying thoughtful words such as 'Good morning', 'We welcome you', and 'Good job today'. The informing action involves providing helpful information such as weather forecasts, the temperature, and accommodation details. Although these actions are very short and can be finished within a single round-trip interaction (e.g. a customer responds with only a 'Good morning' or 'Thank you', and the interaction finishes), they are enough to enhance customer satisfaction with the overall level of service $[12,16,34]$.

Starting a conversation ahead of a customer is important in increasing the number of interactions between a social robot and a customer [27, 32]; hence, the actions were implemented as active behaviours instead of passive 
Fig. 1 Overview of the interaction system employing social robots

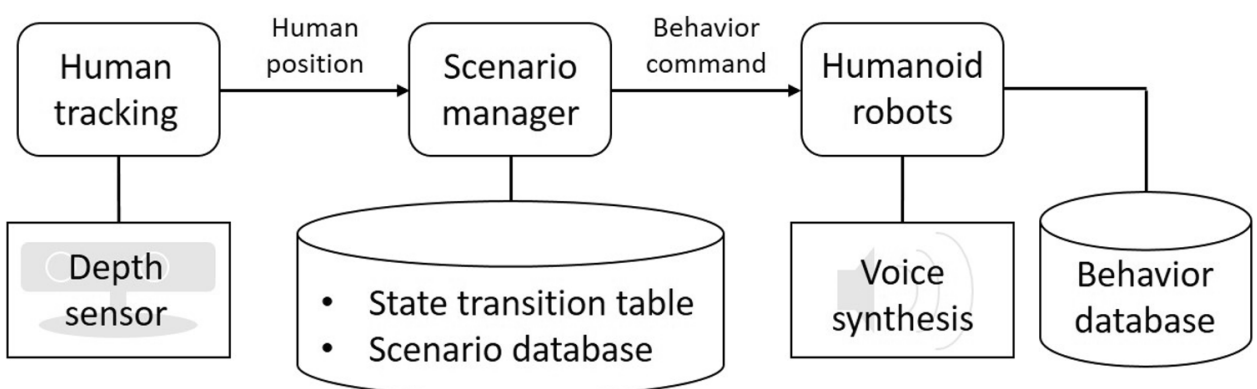

Table 1 State of human position ([x,y]: $x-m$ for corridor, $y-m$ for elevator)

\begin{tabular}{ll}
\hline State of human position & Distance $(d)$ and direction to the robot \\
\hline NON-EXISTENT & $d=$ null \\
APPROACHING & $d>[7.0,6.0]$ with approaching \\
NEAR & $d \leq[3.0,1.0]$ \\
LEAVING & $d>[7.0,6.0]$ with leaving \\
\hline
\end{tabular}

ones. Active behaviour requires detecting a customer's position; thus, a system was developed consisting mainly of two devices (other than a computer): a social robot and a three-dimensional (3D) image sensor capable of recognizing the position of a human. The developed system used desktop-sized (approximately $0.3 \mathrm{~m}$ tall) Sota and CommU (Vstone Co., Ltd.) social robots capable of interacting with humans by voice and through arm, face, and head motions. As the 3D image sensor, the system used a Realsense D435/D415 depth camera (Intel Corp.), which is capable of providing RGB and depth images (maximum range of more than $10 \mathrm{~m}$ ). Human 2D positions were detected from the captured RGB image using the 'OpenPose' algorithm [6], and human 3D positions were computed using the detected 2D positions and the depth image.

Figure 1 shows an overview of our developed system, comprising three components: a human tracking system, a scenario manager, and a humanoid robot. The human tracking system calculates a human's 3D position and their walking direction in the robot coordinate system, using data from the depth sensor; it then sends them to the scenario manager. Based on this information, the scenario manager determines the state of the human position (i.e. 'NON-EXISTENT', 'APPROACHING', 'NEAR', and 'LEAVING'; Table 1). When state transitions occur, the scenario manager selects the proper scenario from the scenario database by referring to a state transition table (Table 2) and then sends a behaviour command (i.e. physical motion and utterance text) to a humanoid robot in accordance with the scenario (e.g. Table 3). A humanoid robot performs the behaviour command
Table 2 State transition table

\begin{tabular}{ll}
\hline State transition & Scenario name \\
\hline NON-EXISTENT $\rightarrow$ APPROACHING & Greeting \\
APPROACHING $\rightarrow$ NEAR & Informing \\
NEAR $\rightarrow$ LEAVING & Farewell \\
LEAVING $\rightarrow$ NON-EXISTENT & Waiting \\
\hline
\end{tabular}

using voice synthesis and a behaviour database. For example, when the robot in the corridor detects a customer approaching it, it would say 'Good morning.' with a bowing behaviour, followed by'It will be warmer today than yesterday.' when the customer walks past it. Finally, when the customer leaves the robots position, the robot will say 'Have a nice day.'

This study prepared two types of heartwarming interaction systems: one using a single robot and one using two robots. The single-robot type is composed of a robot that interacts with passing customers at an entrance or in a corridor. The duration of the interactions with passing customers is short (approximately less than 10 s); hence, the interaction scenario consists of short utterances. When a customer stops in front of a robot, it uses a conversationend word to induce a customer to leave, such as 'Goodbye' or 'Have a good day'. The two-robot type is composed of two robots interacting with a waiting customer at a lobby or in front of an elevator. The length of interactions with waiting customers is often relatively long, such that a single round-trip interaction cannot fill the time; thus, the interaction time is exceeded, unlike when interacting with passing customers.

In this implementation, the two robots consume the excess time in their conversation by including helpful information and recommendations (i.e. promoting the use of the hotel's accommodations) for a customer. Such interactions were implemented because recent studies have shown that conversations between robots can help smooth lengthier human-agent interactions $[3,13]$. They repeat their conversation until a customer leaves. For each system, scenarios are prepared for each time of day (i.e. morning, afternoon, and evening). 
Table 3 Examples of the scenario detail

\begin{tabular}{|c|c|}
\hline Scenario name & Robots' behaviour \\
\hline Greeting & $\begin{array}{l}\text { Robot(s): say 'Good morning.,' 'Good afternoon., 'Good evening.,' 'We welcome you.,' 'Good job } \\
\text { today., and so on with a bow behaviour }\end{array}$ \\
\hline $\begin{array}{l}\text { Informing } \\
\text { (corridor) }\end{array}$ & $\begin{array}{l}\text { Robot: say 'It will be warmer today than yesterday.,'It is sunny day.,' Wi-Fi password is written on } \\
\text { the card holder of your room key.,' 'The reception desk is open all night., and so on }\end{array}$ \\
\hline $\begin{array}{l}\text { Informing } \\
\text { (elevator hall) }\end{array}$ & $\begin{array}{l}\text { [Example 1] } \\
\text { Robot A: say'I got hungry now.' } \\
\text { Robot B: say 'Hotel's restaurant is open until } 10 \text { p.m.' while looking at a robot A } \\
\text { Robot A: say 'Wow, that's great ...' } \\
\text { [Example 2] } \\
\text { Robot A: say'I want to charge my cell phone.' } \\
\text { Robot B: say 'A customer room has a USB charging port next to the bed.' while looking at a robot A } \\
\text { Robot A: say 'Wow, that's convenient ...' }\end{array}$ \\
\hline Farewell & Robot(s): say 'It's been nice meeting you.,' 'Have a nice day', and so on \\
\hline
\end{tabular}

\subsection{Field study: social robots engage in heartwarming interaction in a public area of a hotel}

In order to test the collaboration design 'Continuous Hospitality with Social Robots', a field study was conducted in which social robots engaged in heartwarming interactions while servicing customers in a hotel which is mainly for business use. To conduct the evaluation, customer impressions of the interaction were collected through questionnaires and interviews. This study was conducted in Tokyo over a 2-week period in late March.

\subsubsection{Experimental environment}

This field study was conducted on the first floor of a hotel. The floor included 18 customer rooms, and participants can enter the floor only by elevator. Customers other than the participants could not enter the floor during the 2-week experiment. Figure 2 contains a floor map indicating where the heartwarming interaction systems were installed. The systems were placed in the middle of a corridor and in an elevator hall. The social robots were placed on a stand $(0.3 \mathrm{~m} \times 0.4 \mathrm{~m} \times 1 \mathrm{~m})$ at each location. A Sota robot was placed in the corridor, and a Sota robot and CommU robot were placed in the elevator hall. A 3D image sensor was mounted on a 2-m pole to monitor the system.

\subsubsection{Procedure}

During the 2-week experiment, customers waiting at a reception desk were randomly selected for the field study. The purpose and content of the field study was explained to customers, and they were asked whether they wanted to participate. After customers gave their informed consent to participate in the experiment, they received a gift certificate equivalent to $5000 \mathrm{JPY}$. For child participants, informed consent was received from both the children and their parents. All participants were assigned to rooms on the first floor. After informed consent, the participants
Fig. 2 Floor map and the position of humanoid robots and sensors

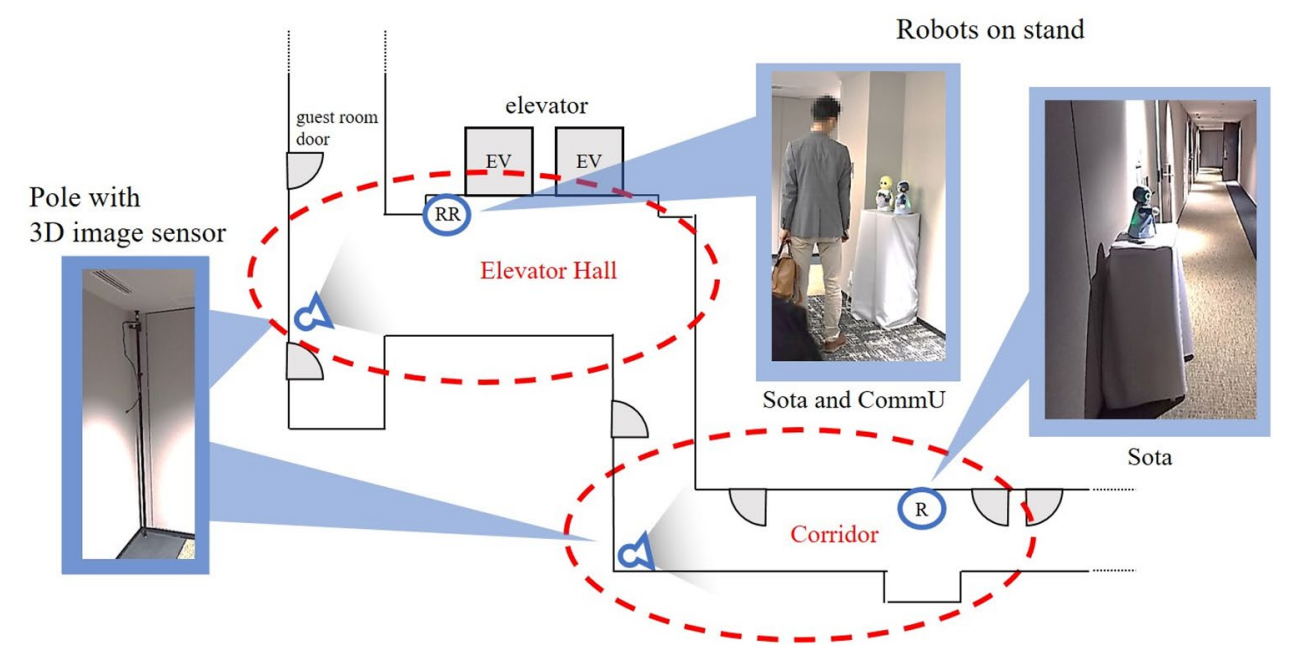


were instructed to use the hotel as usual and submit a completed questionnaire to us at checkout. The participants would interact with the systems when leaving from and returning to the first floor. In addition, they were asked to participate in an interview at checkout.

\subsubsection{Participants}

A total of 67 Japanese customers participated in the field study; their ages ranged from teenage to over 60 years. Sixty-four questionnaire sheets were collected, 11 of which did not have answers to several items. Accordingly, 53 sheets were used for the analysis. A single customer is defined herein as a customer who stays alone, while a group customer is defined as a customer who stays with family or friends. Thirteen females and 11 males were single customers, while 16 females and 13 males were group customers. The participants stayed at the hotel from one to six nights.

\subsubsection{Measurement and analysis}

Using a questionnaire, customer impressions of the heartwarming interaction with social robots were collected (Table 4). Items R1-6 are an evaluation of each robot system ( $\mathrm{R} 3$ is an evaluation for Q1). Items $\mathrm{H} 1-4$ are an evaluation of customer satisfaction with the hotel to answer Q2. The items are important aspects of customer satisfaction [19, 22]. Item R6 is an evaluation for Q5. Only those who listened to the robots' recommendations could answer the item. Items $\mathrm{P} 1-3$ are an investigation into the personal attributes of the participants. A 7-point Likert scale was used to evaluate items R1-6 and H1-4 (R1: 1: strongly disagree, 4: no opinion, 7: strongly agree; R5: 1 : became much worse, 4: no change, and 7: became much better; and R2-4, R6, H1-4: 1: completely disagree, 2: mostly disagree, 3: slightly agree, 4: moderately agree, 5: strongly agree, 6 : very strongly agree, and 7 : completely agree). A multiple choice form was used for items P1-3 (P1: 1: this is the first time, 2: less than once a week, 3: less than once a month, 4: less than once a week, and 5: more than once a week; and P2, P3: 1: this is the first time, 2: occasionally, 3: do so at fixed intervals, and 4: do so every day).

A positive answer rate was calculated for items R1-6 and $\mathrm{H} 1-4$. A positive answer is defined as follows: $\mathrm{R} 1, \mathrm{R} 5$ : $\geq 5 ; R 2: \leq 2 ;$ and $R 3, R 4, R 6$, and $H 1-4: \geq 3$. They are answers that indicate at least a moderate positive effect.

For the exploratory investigation, a three-way (single or two robots, male or female, single or group customers) analysis of variance (ANOVA) was used for each item in order to answer Q3. A Spearman's rank correlation coefficient was calculated between the questionnaire items and the personal attributes of the participants (i.e. the number of nights, frequency of staying at the hotel, and degree of interaction with social robots and the voice-controlled speaker). The test for non-correlation was used to answer Q4.

In an interview, the participants were asked about the system's merits and demerits, and their expectations of the system. Employees' impressions and awareness about this field test were also gathered.

Table 4 Items in the questionnaire

\begin{tabular}{|c|c|}
\hline Item & Question \\
\hline \multicolumn{2}{|c|}{ Impression of robots } \\
\hline $\mathrm{R} 1(x)$ & Should $x$ be placed at a hotel? \\
\hline $\mathrm{R} 2(x)$ & Did your interaction with $x$ feel intrusive? \\
\hline $\mathrm{R} 3(x)$ & Did you feel warm from interaction with $x$ ? \\
\hline $\mathrm{R} 4(x)$ & Did you enjoy interaction with $x ?$ \\
\hline $\mathrm{R} 5(x)$ & How did your mood change by interaction with $x ?$ \\
\hline R6 & Did you think of following information recommended from robots at an elevator hall? \\
\hline \multicolumn{2}{|c|}{$x=c:$ a robot in a corridor, $e$ : robots in an elevator hall } \\
\hline \multicolumn{2}{|c|}{ Evaluation for hospitality } \\
\hline $\mathrm{H} 1$ & By social robots' service, did you feel Japanese-style hospitality from the hotel? \\
\hline $\mathrm{H} 2$ & By social robots' service, did you feel comfort during your stay? \\
\hline $\mathrm{H} 3$ & By social robots' service, are you compelled to recommend others to stay here? \\
\hline $\mathrm{H} 4$ & By social robots' service, are you likely to stay here again? \\
\hline \multicolumn{2}{|c|}{ Personal attribution } \\
\hline P1 & How often do you use a business hotel? \\
\hline $\mathrm{P} 2$ & How much have you interacted with a social robot? \\
\hline P3 & How much have you verbally interacted with a voice-controlled speaker? \\
\hline
\end{tabular}


Three coders and an experimenter analysed the video data to identify the number and type of interactions that the participants had with the robots. Two coders were assigned to the robot in the corridor, and the other coder and the experimenter were assigned to the robots in the elevator hall. They counted customers who passed near the robots in the video data, and judged whether or not customers ignored the robots. An analysis of the overlapped data (ten percent of the all video data in each case) were well matched (counting passing customers: a corridor Cohen's Kappa $K=0.86$, an elevator hall $K=0.76$; judgement of ignoring: a corridor $K=0.82$, an elevator hall $K=1$ ).

\subsubsection{Results}

Figure 3 shows the rates of each answer for each questionnaire item. As for the evaluation of each robot system (i.e. items R1-6), more than 70\% of the participants had positive impressions of each system without item R1. In addition, more than approximately $70 \%$ of the participants had positive impressions of their satisfaction with the hotel (i.e. items $\mathrm{H} 1-4)$. Item R6 was completed by 37 participants.

Several main and interaction effects were identified using the three-way ANOVA of the questionnaire (Fig. 4). A significant main effect, gender, was found in item R1. Compared to males, females more strongly agree that the heartwarming interaction with social robots should be used at a hotel $(F(1,49)=4.94, p<.05$, ES : $r=.30)$. Significant interaction effects were found in item R3. Compared to single male customers, single female customers experience more warmth from the interaction with social robots $(F(1,22)=5.85, p<.05, \mathrm{ES}: r=.46)$. With the two robots in the elevator hall, a female also experiences more warmth than a male $(F(1,49)=4.32, p<.05$, ES $: r=.29)$. Male customers feel more warmth from the interaction with the single robot in the corridor than with the two robots in the elevator hall $(F(1,22)=6.05, p<.05$, ES : $r=.46)$. A significant interaction effect was also found in item R4. Females enjoy interaction with the two robots in the elevator hall more than with the single robot in the corridor $(F(1,49)=5.14, p<.05, \mathrm{ES}: r=.31)$.

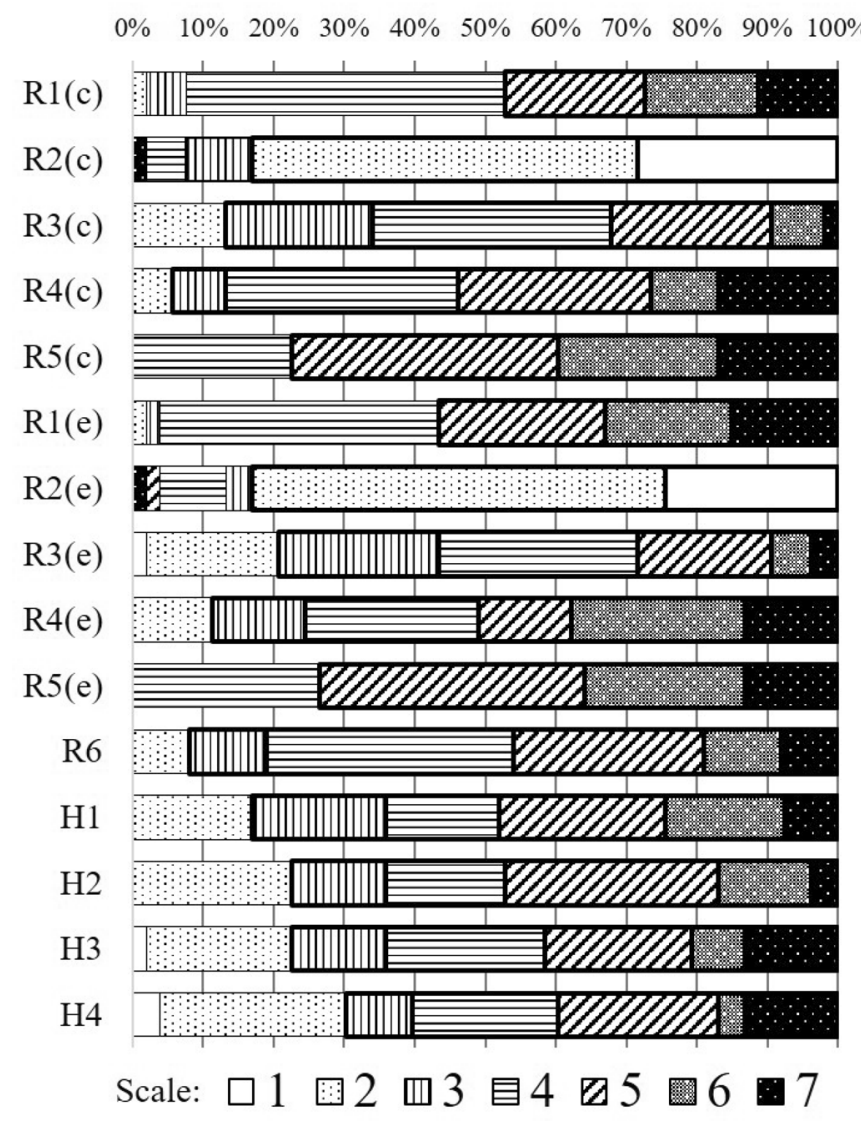

\begin{tabular}{|c|c|c|}
\hline $\begin{array}{c}\text { Positive } \\
\text { answer } \\
\text { rate (\%) }\end{array}$ & $\begin{array}{c}\text { Score } \\
\text { average }\end{array}$ & $\begin{array}{c}\text { Standard } \\
\text { deviation }\end{array}$ \\
\hline 47.2 & 4.76 & 1.24 \\
\hline 83.0 & 2.02 & 0.73 \\
\hline 86.8 & 3.96 & 1.52 \\
\hline 94.3 & 4.78 & 1.75 \\
\hline 77.4 & 5.34 & 1.00 \\
\hline 56.6 & 4.99 & 1.33 \\
\hline 83.0 & 2.13 & 0.88 \\
\hline 79.3 & 3.76 & 1.86 \\
\hline 88.7 & 4.66 & 2.25 \\
\hline 73.6 & 5.23 & 0.99 \\
\hline 91.9 & 4.46 & 1.49 \\
\hline 83.0 & 4.27 & 2.06 \\
\hline 77.4 & 4.09 & 1.87 \\
\hline 77.4 & 4.15 & 2.63 \\
\hline 69.8 & 3.96 & 2.70 \\
\hline
\end{tabular}

Fig. 3 Rates and average of each answer in the questionnaire (the bold portions denote positive answers) 
Fig. 4 Average and standard error of questionnaire items for each attribution (analysis of variance, ${ }^{*} p<.05$ )

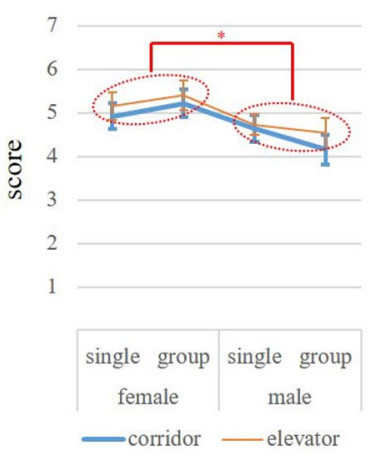

R1: Necessity

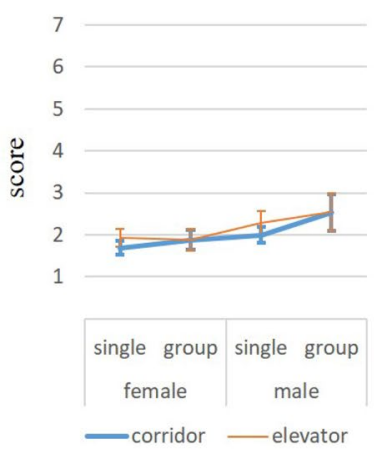

R2: Intrusiveness

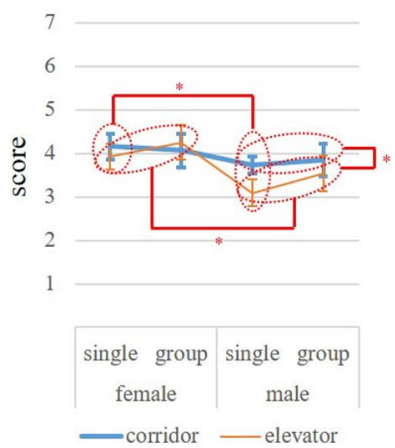

R3: Warmth

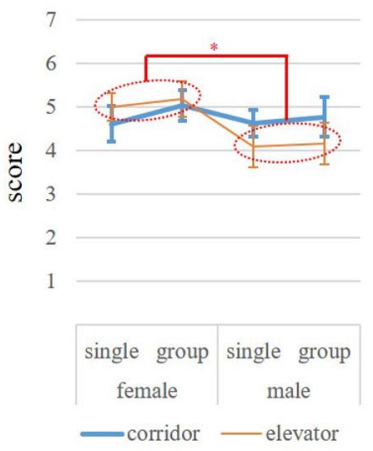

R4: Enjoyment

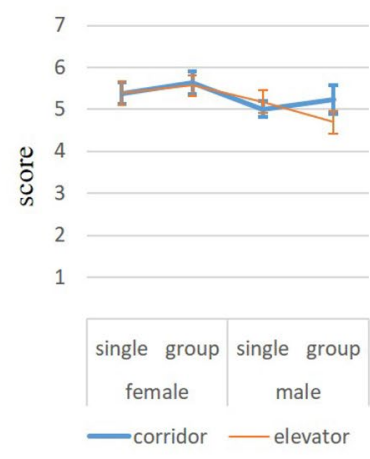

R5: Mood
Table 5 shows the correlation coefficients between the questionnaire items and the individual attributes of the participants. Significant weak correlations were found between them. The number of nights was weakly correlated with items $\mathrm{R} 3(\mathrm{c})$ and $\mathrm{R} 5(\mathrm{e})$. A longer stay at the hotel made the customer feel more warmth from interactions with the single robot $(S=17533, p<.05)$ and caused the customer's mood to be enhanced more by two robots $(S=17379, p<.05)$. The degree of interaction with social robots is weakly correlated with item
Table 5 Correlation coefficients between the questionnaire items and the personal attributes of the participants

\begin{tabular}{lllll}
\hline Item & Number of nights & $\begin{array}{l}\text { Frequency of } \\
\text { staying at hotel }\end{array}$ & $\begin{array}{l}\text { Experience of interac- } \\
\text { tion with a social robot }\end{array}$ & $\begin{array}{l}\text { Experience of interaction } \\
\text { with a voice-controlled } \\
\text { speaker }\end{array}$ \\
\hline R1(c) & 0.239 & 0.0442 & -0.117 & -0.0488 \\
R2(c) & 0.0212 & 0.0838 & 0.198 & $\mathbf{0 . 2 8 9 *}$ \\
R3(c) & $\mathbf{0 . 2 9 3 ^ { * }}$ & -0.0203 & $-\mathbf{0 . 2 9 7 ^ { * }}$ & -0.225 \\
R4(c) & 0.204 & -0.0347 & -0.191 & -0.0904 \\
R5(c) & 0.124 & -0.119 & -0.0571 & -0.207 \\
R1(e) & 0.158 & 0.116 & -0.0612 & -0.0647 \\
R2(e) & 0.267 & 0.135 & 0.189 & $\mathbf{0 . 3 8 0 *}$ \\
R3(e) & 0.267 & -0.141 & -0.0189 & -0.159 \\
R4(e) & 0.138 & -0.0919 & -0.00127 & -0.254 \\
R5(e) & $\mathbf{0 . 2 9 9 *}$ & -0.101 & -0.205 & -0.146 \\
H1 & 0.211 & -0.0947 & -0.0254 & -0.147 \\
H2 & 0.267 & -0.0771 & -0.0480 & -0.133 \\
H3 & 0.146 & -0.0475 & 0.00596 & -0.172 \\
H4 & 0.177 & -0.00739 & -0.0540 & -0.183 \\
\hline
\end{tabular}

Spearman's rank correlation coefficient; test for non-correlation, ${ }^{*} p<.05$ 
R3(c). Those engaging in more interaction with social robots felt less warmth from interaction with the single robot $(S=32175, p<.05)$. The degree of interaction with a voice-controlled speaker was weakly correlated with items R2(c) and R2(e). Those who interacted with a voice-controlled speaker felt more intrusion from interactions with the single robot and the two robots $(S=17624, p<.05 ; S=15381, p<.01)$.

Table 6 shows a list of interview answers ( 23 females and 12 males), through which many comments about merits and expectations were obtained. Some participants left several comments over the categories, and others left comments about only one category. It is noted that the male interviewees provided less comments overall than the female did. As for the merits, many females mentioned positive emotional effects of interactions with social robots (e.g. felt interesting, enjoyable, relieved, non-lonely, brighter, and warm). As for the expectations, participants hoped to talk more with the robots about various things. In addition, we interviewed two members of the hotel's concierge staff that helped us manage the experiment. They provided us with insight into the effects of the robots from the perspective of the hotel staff. Their comments are largely similar to those reported by the customer participants. In particular, the employees reported that they also felt the benefits of the heartwarming interactions with the social robots. Only unique one of them is that the housekeeping employees were pleased with the heartwarming interaction with robots, which they strongly argued with surprise.

The total number of interaction opportunities (i.e. when a customer passed near the robot) between a customer and a robot was 439 in the corridor, and 946 in the elevator hall. Since some customers were assigned to a room where they would not need to pass by the corridor robot, there were less interaction opportunities in the corridor than in the elevator hall. Participants were found to ignore the robot in $12.5 \%$ of the interaction opportunities in the corridor and $26.0 \%$ in the elevator hall.

\section{Discussion}

\subsection{Impact of heartwarming interaction with social robots at a hotel}

Most participants admitted experiencing at least a slight warmth from the interaction with robots (i.e. items R3). Thus, as an answer for Q1, it was shown that interactions with social robots can provide a heartwarming experience for hotel customers. The results of interviews also support this, because many participants mentioned feeling relieved, less lonely, brighter, and warm from the interactions with social robots. Most participants also admitted

Table 6 List of comments in the interview with customer participants

\begin{tabular}{|c|c|c|}
\hline Category & Comment & $\begin{array}{l}\text { Number } \\
\text { (females, } \\
\text { males) }\end{array}$ \\
\hline \multirow[t]{8}{*}{ Merits } & Feel cute, interesting, or enjoyable particularly for conversation of two robots at an elevator hall & $13(11,2)$ \\
\hline & Feel relieved, non-lonely, brighter, or warm for robots & $8(7,1)$ \\
\hline & Greeting is nice & $4(3,1)$ \\
\hline & Provided information is useful for me & $3(3,0)$ \\
\hline & It is nice that robots' talk is different in hours and stops at midnight & $2(1,1)$ \\
\hline & Robots trigger a conversation with others & $2(2,0)$ \\
\hline & Small robots are not more intrusive than a human & $2(1,1)$ \\
\hline & Robots may be used for crime prevention & $2(1,1)$ \\
\hline Demerits & Robots seem to be noisy for a room near them & $2(1,1)$ \\
\hline \multirow[t]{10}{*}{ Expectation } & More robot responses when talking to them & $21(15,6)$ \\
\hline & More various useful information (guide to a room and around a hotel and current time) & $9(8,1)$ \\
\hline & More various conversations of two robots at an elevator hall & $6(3,3)$ \\
\hline & Smoother conversation (e.g. listenability, responsiveness, and adaptability to a situation) & $6(4,2)$ \\
\hline & Better arrangement (difficult to find, far from my room) \&1 $(1,0)$ & $1(1,0)$ \\
\hline & Want robots also in a room & $1(0,1)$ \\
\hline & English communication & $1(1,0)$ \\
\hline & Individual recognition & $1(1,0)$ \\
\hline & Better cloth design & $1(1,0)$ \\
\hline & Replace CommU with Sota (CommU is scary) & $1(1,0)$ \\
\hline
\end{tabular}


experiencing at least a slightly positive effect from the interaction with robots on items $\mathrm{R} 2,4$, and 5 (i.e. non-intrusive, enjoyable, and mood). Thus, the system is a beneficial service for many customers from various aspects. On the other hand, the result of item R1 indicated that half of the participants did not need the system at the hotel, although they admitted a positive effect from it. This can be attributed to the service being regarded as a valueadded service; that is, it depends on individual demand.

Most participants also admitted experiencing at least a slightly positive effect from the interaction with robots on items $\mathrm{H} 1-4$ (i.e. Japanese-style hospitality, comfort, making recommendations, and repeated use). Thus, as an answer for Q2, it was shown that interactions with a social robot can enhance a customer's satisfaction with the overall service at a hotel. The results of the interview, where there were many positive comments, provide further evidence for this finding. However, the current study is limited by the fact that all of our participants were exposed to the social robots, and future work should be conducted to evaluate this question through the use of a controlled experiment.

The findings from the field study indicate that a social robot can engage in a heartwarming interaction that enhances hospitality in a hotel environment, using the 'Continuous Hospitality with Social Robots' collaboration design. This fact could be applied to other fields such as retail, restaurants, and nursing. However, there are several limitations for their implementations. First, cultural and situational factors should be considered. Different situations will require different manners of interaction. Second, the effects of a robot's appearance should also be investigated, by determining whether its traits are humanlike/ robot-like/animal-like, and by examining the effects of any physical body parts. If a different robot had been used in this study, the results may have been different. Moreover, the personality of customers is also an important factor which determines their impression of the system. In this field study, participants who were friendly to robots may have been predominant.

The heartwarming interaction system has significant room for improvement as the interview results provide us with clues to improve the system. In fact, some participants could not obtain positive effects from the system. The main comments on expectations indicate that participants hoped the robot would respond to their statements and provide other various useful information and conversations. When employing the designed functions and content, the system could enhance customer satisfaction mostly in terms of hotel service. Moreover, an undermentioned exploratory investigation suggests several possible impacts resulting from differences in personal and situational attributes; that is, it is important to provide an interaction that correlates with the individual attributes of customers. Accordingly, individual recognition technology might be an important key to an improved interaction system.

\subsection{Effect of personal and situational attributes on impressions of heartwarming interaction with robots}

The exploratory investigation suggested possible effect differences resulting from differences in personal and situational attributes. Regarding Q3, the results of the three-way ANOVA suggested differences related to gender. First, males did not value the warmth and enjoyment provided by the two robots in the elevator hall as highly as the females did. In other words, the conversation between the two robots was not an effective interaction for a male. It was inferred that the reason for this was that males generally prefer silence [10], which was also supported by the interview results showing that many female participants found the robots' conversation to be cute, interesting, or enjoyable. It is noted that only a few males felt that way although gender imbalance of interviewees has to be taken into consideration. Thus, it may be preferable to avoid providing excessive interaction with male hotel customers. Second, compared to males, females staying by themselves tended to feel warmth from the interaction with social robots. The warmth felt by single customers seemed to alleviate their sense of loneliness. Considering the interview results showing that some females and only one male participant felt less lonely through the interaction with social robots, it was inferred that the reason for this was that females have greater awareness of their own loneliness. Thus, it is clear that the interaction should be more aggressively provided to single female customers at hotels. It is noted that some studies claim that males simply cannot admit to feeling lonely $[5,9]$. Third, females tend to more strongly agree that the heartwarming interaction system should be made available at hotels. It was inferred that females could more readily understand the value of the system because of the tendencies mentioned above.

Regarding Q4, the correlation results suggest an important tendency in long-term interactions with social robots at a hotel. First, significant weak positive correlations were found between the numbers of nights stayed and two items: R3(c) and R5(e). A longer stay allows participants to have more contact with robots; hence, it is inferred that these positive correlations indicate an accumulation of the robots' main contributions. Accordingly, it is concluded that the single robot in the corridor generated additional warmth and that the two robots in the elevator hall contributed to enhancing the 
customers' mood. The number of nights stayed was also positively correlated with most of the other items; thus, it is inferred that providing more interaction can generate more positive benefits for a hotel. Second, a significant weak negative correlation was found between the degree of interaction with social robots and item R3(c). Observing the interactions between humans and social robots reveals information about the robots' abilities in terms of recognition, physical movement, and communication skill. A single robot in a corridor only engages in short conversations, unlike two robots in an elevator hall and general social robots; hence, this gap in robotic functionality might cause experienced participants to perceive a lack of warmth. Additionally, significant weak positive correlations were found between the degree of interaction with the voice-controlled speaker and items R2(c) and R2(e). It was inferred that this occurred because interaction with the voice-controlled speaker reduced interest in verbal communication with robots. A similar tendency appears in other studies, in which students tended to gradually lose interest in interaction with social robots during long-term experiments [17, 18]. Thus, when a robot talks to someone uninterested in verbal communication with a robot, the communication would be perceived as intrusive. The same tendency was found in the correlation between the degree of interaction with a social robot and items R2(c) and $\mathrm{R} 2(\mathrm{e})$, suggesting that greater experience with robots leads to more negative responses by the participant. The present generation will have many verbal interactions with robots or voice-controlled speakers in the near future; thus, attention must be paid to the negative aspects of heartwarming interaction provided by social robots. One possible explanation of this relationship between greater experience with negative responses is that individuals who have used voice assistants and robots in the past perceive them as tools rather than entities that have intention. To achieve the feeling of the heartwarming interactions we focused on in this paper, it is important to imagine the conversation partner's intention. For example, to create interpersonal warmth when being greeted, you must imagine that the partner's intention is to welcome you and to establish a good relationship. However, if the individual regards the robot as a tool, they may not perceive that the robot's intention as described above, and then greeting will become just text information. Since experienced individuals are likely to understand that the robot does not have intention, this can lead to the negative responses and the lack of heartwarming interactions. Finally, note that this repeated testing may cause type I error; hence, reverification is likely required.

\subsection{Recommendation in heartwarming interaction}

Regarding Q5, interestingly, almost all participants considered following the recommendations from the two robots in the elevator hall. This tendency indicates the potential of using social robots to provide advertising, in addition to providing heartwarming interaction. In advertising research, warmth is an important aspect in improving sales totals through commercials [1]. That is, conveying warmth to a customer induces them to react positively to the provider's advertisement. Hence, our field study shows that heartwarming interaction with a social robot might induce a customer to follow recommendations from the robot. Future research will aim to verify whether human-agent interactions can provide an effective means of advertising.

\subsection{Impact of robots' heartwarming interaction with housekeeping employees}

Employees' comments revealed an unexpected aspect of the interaction with social robots: housekeeping employees seemed to benefit from heartwarming interaction with robots. These employees clean customer rooms and public areas during the daytime. During that time, they had several opportunities to interact with robots in public areas of the hotel. It is inferred that the housekeeping employees may lack social approval and self-esteem because they engage in behind-the-scenes work, in which they provide service for customers but rarely interact with them face-to-face. In other words, they have little chance to be appreciated by customers, unlike in general service encounter work. In particular, tipping is not customary for hotel cleaning in Japan. Thus, the lack of personal interaction experienced by housecleaning employees might cause them to lack social approval and self-esteem; thus, the robots might benefit these employees through heartwarming interaction.

The above conjecture suggests a novel application of heartwarming interaction; that is, heartwarming interaction supporting the mental well-being of workers engaged in behind-the-scenes work. Because heightened social approval and self-esteem enhance motivation to work [30], heartwarming interaction would be beneficial in terms of employee management. In circumstances in which collaborative work with robots has increased, partnerships between robots and employees should receive greater focus in future research.

\section{Conclusion}

To explore the possibility that social robots can engage in heartwarming interaction, a prototype employing social robots was developed, and a field study in which 
robots provided heartwarming interaction for hotel customers was conducted, using the 'Continuous Hospitality with Social Robots' collaboration design. After collecting and analysing customer impressions of the interaction by questionnaire, it was found that social robots' ability to provide heartwarming interaction enhances customers' overall satisfaction with the hotel services.

This exploratory investigation suggested possible impacts caused by differing personal and situational attributes. First, results show that a heartwarming interaction satisfies females more than it satisfies males. In particular for males, conversations between two robots are relatively ineffective as an interaction service. Second, the contribution of the heartwarming interaction can accumulate day by day and increase customer satisfaction. In more detail, the single robot in the corridor contributed a relatively high degree of warmth, while the two robots in the elevator hall contributed more towards enhancing the customers' mood. Third, the experience of verbal interaction with a robot or a voice-controlled speaker negatively affects the evaluation. People will have many experiences engaging in verbal interaction with technology in the near future; hence, it is important to continually evaluate the negative aspects of heartwarming interaction provided by social robots.

Finally, other applications of the proposed system were examined, namely effective advertising through heartwarming interactions and mental support to behind-the-scenes employees.

Funding This study was conducted in cooperation with Tokyu Stay Co., Ltd., and Tokyu Fudosan R\&D Center Inc. They provided a hotel for the field test and monetary support. This study is also monetarily supported by a Grant-in-Aid for Research Activity Start-up, JSPS KAKENHI Grant Number 18H06479.

\section{Compliance with ethical standards}

Conflict of interest Yuichiro Yoshikawa and Hiroshi Ishiguro consulted for Vstone Co., Ltd., which sells CommU and Sota, and received compensation. Hiroshi Ishiguro also owns stock in the company.

Ethical approval This study involved human participants and was approved by the ethics committee of the Graduate School of Engineering Science at Osaka University (No. 29-14). We obtained informed consent from participants.

\section{References}

1. Aaker DA, Stayman DM, Hagerty MR (1986) Warmth in advertising: measurement, impact, and sequence effects. J Consum Res 12(4):365-381

\section{SN Applied Sciences}

2. Ariffin AAM, Maghzi A (2012) A preliminary study on customer expectations of hotel hospitality: influences of personal and hotel factors. Int J Hosp Manag 31 (1):191-198

3. Arimoto T, Yoshikawa Y, Ishiguro H (2018) Multiple-robot conversational patterns for concealing incoherent responses. Int J Soc Robot 10(5):583-593

4. Bayes MA (1972) Behavioral cues of interpersonal warmth. J Consult Clin Psychol 39(2):333-339

5. Borys S, Perlman D (1985) Gender differences in loneliness. Pers Soc Psychol Bull 11(1):63-74

6. Cao Z, Simon T, Wei SE, Sheikh Y (2017) Realtime multi-person $2 \mathrm{~d}$ pose estimation using part affinity fields. In: Conference on computer vision and pattern recognition (CVPR), Honolulu, 2017, IEEE, Proceedings, pp 1302-1310

7. Chung MJ, Cakmak M (2018) "How was your stay?": exploring the use of robots for gathering customer feedback in the hospitality industry. In: 27th international symposium on robot and human interactive communication (RO-MAN), Nanjing, 2018, IEEE, Proceedings, pp 947-954

8. Chung-En Y (2018) Humanlike robot and human staff in service: age and gender differences in perceiving smiling behaviors. In: 7th international conference on industrial technology and management (ICITM), Oxford, 2018, IEEE, Proceedings, pp 99-103

9. Cramer KM, Neyedley KA (1998) Sex differences in loneliness: the role of masculinity and femininity. Sex Roles 38(7-8):645-653

10. DeFrancisco VL (1991) The sounds of silence: how men silence women in marital relations. Discourse Soc 2(4):413-423

11. Fiske ST, Cuddy AJ, Glick P (2007) Universal dimensions of social cognition: warmth and competence. Trends Cogn Sci 11(2):77-83

12. Frazer Winsted $K$ (2000) Service behaviors that lead to satisfied customers. Eur J Mark 34(3-4):399-417

13. lio T, Yoshikawa Y, Ishiguro H (2016) Pre-scheduled turn-taking between robots to make conversation coherent. In: The 4th international conference on human-agent interaction, Singapore, 2016, ACM, Proceedings, pp 19-25

14. Ivanov SH, Webster C (2017) Adoption of robots, artificial intelligence and service automation by travel, tourism and hospitality companies-a cost-benefit analysis. In: International scientific conference "contemporary tourism-traditions and innovations, Sofia, 2017. https://s3.amazonaws.com/academia.edu. documents/53959355/Adoption_of_robots_by_tourist_compa nies_-_a_CBA_-_full_text.pdf?AWSAccessKeyld = AKIAIWOWYY GZ2Y53UL3A\&Expires $=1549002644 \&$ Signature $=$ QpnF0eS0td qGyf8lqBzhUavCNrs\%3D\&response-content-disposition = inlin e\%3B\%20filename\%3DADOPTION_OF_ROBOTS_ARTIFICIAL INTELLIGEN.pdf. Accessed 1 Feb 2019

15. Ivanov SH, Webster C, Berezina K (2017) Adoption of robots and service automation by tourism and hospitality companies. Revista Turismo \& Desenvolvimento 27(28):1501-1517

16. Kattara HS, Weheba D, El-Said OA (2008) The impact of employee behaviour on customers' service quality perceptions and overall satisfaction. Tour Hosp Res 8(4):309-323

17. Kanda T, Sato R, Saiwaki N, Ishiguro H (2007) A two-month field trial in an elementary school for long-term human-robot interaction. IEEE Trans Robot 23(5):962-971

18. Komatsubara T, Shiomi M, Kanda T, Ishiguro H, Hagita N (2014) Can a social robot help children's understanding of science in classrooms?. In: The 2nd international conference on humanagent interaction, Tsukuba, 2014, ACM, Proceedings, pp 83-90

19. Lemmink J, Mattsson J (1998) Warmth during non-productive retail encounters: the hidden side of productivity. Int J Res Mark 15(5):505-517

20. Lemmink J, Mattsson J (2002) Employee behavior, feelings of warmth and customer perception in service encounters. Int J Retail Distrib Manag 30(1):18-33 
21. Li J, Canziani BF, Barbieri C (2018) Emotional labor in hospitality: positive affective displays in service encounters. Tour Hosp Res 18(2):242-253

22. Lloyd AE, Luk ST (2011) Interaction behaviors leading to comfort in the service encounter. J Serv Mark 25(3):176-189

23. Morishita S (2016) Managing omotenashi in onsen ryokans: a case study of Kurokawa Onsen in Kyushu. J Glob Tour Res 1(2):157-160

24. Nieto D, Quesada-Arencibia A, García CR, Moreno-Díaz R (2014) A social robot in a tourist environment. In: Hervás $R$, Lee $S$, Nugent C, Bravo J (eds) Ubiquitous computing and ambient intelligence: personalisation and user adapted services: 8th international conference, Belfast, 2014, Proceedings, vol 8867. Springer, Berlin, pp 21-24

25. Osawa H, Ema A, Hattori H, Akiya N, Kanzaki N, Kubo A, Koyama T, Ichise R (2017) Analysis of robot hotel: Reconstruction of works with robots. In: 26th international symposium on robot and human interactive communication (RO-MAN), Lisbon, 2018, IEEE, Proceedings, pp 947-954, 2018

26. Pan Y, Okada H, Uchiyama T, Suzuki K (2013) Direct and indirect social robot interactions in a hotel public space. In: International conference on robotics and biomimetics (ROBIO), Shenzhen, 2013, IEEE, Proceedings, pp 1881-1886

27. Pan Y, Okada H, Uchiyama T, Suzuki K (2015) On the reaction to Robot's speech in a hotel public space. Int J Soc Robot 7(5):911-920

28. Pinillos R, Marcos S, Feliz R, Zalama E, Gómez-García-Bermejo J (2016) Long-term assessment of a service robot in a hotel environment. Robot Auton Syst 79:40-57

29. Primawati $S$ (2018) The role of artificially intelligent robot in the hotel industry as a service innovation. In: Proceedings of ENTER2018 Ph.D. workshop, vol 42

30. Pyszczynski T, Greenberg J, Solomon S, Arndt J, Schimel J (2004) Why do people need self-esteem? A theoretical and empirical review. Psychol Bull 130(3):435-468

31. Reuland R, Choudry J, Fagel A (1985) Research in the field of hospitality. Int J Hosp Manag 4(4):141-146

32. Rodriguez-Lizundia E, Marcos S, Zalama E, Gómez-García-Bermejo J, Gordaliza A (2015) A bellboy robot: study of the effects of robot behaviour on user engagement and comfort. Int J Hum Comput Stud 82:83-95

33. Shani A, Uriely N, Reichel A, Ginsburg L (2014) Emotional labor in the hospitality industry: the influence of contextual factors. Int J Hosp Manag 37:150-158

34. Shaw Brown C, Sulzer-Azaroff B (1994) An assessment of the relationship between customer satisfaction and service friendliness. J Organ Behav Manag 14(2):55-76

35. Sundaram DS, Webster C (2000) The role of nonverbal communication in service encounters. J Serv Mark 14(5):378-391

36. Tanaka K, Yamashita N, Nakanishi H, Ishiguro H (2016) Teleoperated or autonomous?: How to produce a robot operator's pseudo presence in HRI. In: The 11th international conference on human-robot interaction (HRI), Christchurch, 2016, IEEE Press, Proceedings, pp 133-140

37. Trovato G, Zecca M, Sessa S, Jamone L, Ham J, Hashimoto K, Takanishi A (2013) Cross-cultural study on human-robot greeting interaction: acceptance and discomfort by Egyptians and Japanese. J Behav Robot 4(2):83-93

38. Tung VWS, Au N (2018) Exploring customer experiences with robotics in hospitality. Int J Contemp Hosp Manag 30(7):2680-2697

39. Tung VWS, Law R (2017) The potential for tourism and hospitality experience research in human-robot interactions. Int J Contemp Hosp Manag 29(10):2498-2513

40. Zalama E, García-Bermejo JG, Marcos S, Domínguez S, Feliz R, Pinillos R, López J (2014) Sacarino, a service robot in a hotel environment. In: Armada MA, Sanfeliu A, Ferre M (eds) ROBOT2013: first iberian robotics conference, Madrid, 2013, vol 253. Springer, Cham, pp 3-14

Publisher's Note Springer Nature remains neutral with regard to jurisdictional claims in published maps and institutional affiliations. 